\title{
Effects of Clinical Characteristics on Sleep Quality in Patients with Chronic Temporomandibular Disorders
}

\author{
Yeon-Hee Lee1 ${ }^{1,}$, Q-Schick Auh ${ }^{1}$, Jung-Sub An ${ }^{2}$, and Tae Kim ${ }^{3}$ \\ 1 Department of Orofacial Pain and Oral Medicine, Kyung Hee University Dental Hospital, Seoul, Korea; \\ omod0209@gmail.com, dental21@khu.ac.kr \\ 2 Department of Orthodontics, Seoul National University Dental Hospital, Seoul, Korea; \\ maki111@hanmail.net \\ 3 Department of Biomedical Science and Engineering, Gwangju Institute of Science and Technology, \\ Gwangju, Korea; tae-kim@gist.ac.kr \\ * Correspondence: omod0209@gmail.com; Tel.: Phone: 82-2-958-9454; Fax: 82-2-962-8124
}

\begin{abstract}
Objectives: This study aimed to investigate and compare sleep quality between patients with chronic temporomandibular disorder and healthy controls, and to analyze the association of sleep quality with disease characteristics, obstructive sleep apnea risk factors, and excessive daytime sleepiness. Methods: Chronic temporomandibular disorder patients ( $\mathrm{n}=503)$ and 180 age- and sexmatched healthy controls were included, who completed well-organized clinical report and answered questions on sleep quality (Pittsburgh Sleep Quality Index), sleep apnea risk factors (STOPBang questionnaire), and excessive daytime sleepiness (Epworth sleepiness scale). Results: Mean global Pittsburgh Sleep Quality Index scores were significantly higher in the patients $(6.25 \pm 2.77)$ than in healthy controls $(6.25 \pm 2.77)(\mathrm{p}<0.001)$. Poor sleep was significantly more prevalent in the patient group $(56.9 \%)$ than in healthy controls $(22.2 \%)(\mathrm{p}<0.001)$. Compared with healthy controls, chronic temporomandibular disorder patients had a higher likelihood of obstructive sleep apnea. (STOP-Bang total score $\geq 3 ; 7.2 \%$ vs. $16.1 \%$; $\mathrm{p}<0.01$ ) and higher excessive daytime sleepiness (Epworth sleepiness scale score $\geq 10 ; 12.8 \%$ vs. $19.7 \%$; $\mathrm{p}<0.05$ ). Age (odds ratio $=2.551 ; \mathrm{p}<0.001$ ), female sex (odds ratio $=1.885 ; \mathrm{p}=0.007$ ), total Epworth sleepiness scale score (odds ratio $=1.839 ; \mathrm{p}=0.014$ ), and headache attributed to temporomandibular disorder (odds ratio $=1.519 ; \mathrm{p}=0.049$ ) were the most powerful predictors of poor sleep (global Pittsburgh Sleep Quality Index score $\geq 5$ ) in chronic temporomandibular disorder patients. Conclusion: Chronic temporomandibular disorder patients had sleep quality impairment. Various factors, including peripheral and central factors, affect the patient's sleep quality. Therefore, in addition to sleep quality and sleep-related problems, the underlying central mechanism for poor sleep quality should be assessed when treating chronic temporomandibular disorder patients.
\end{abstract}

Keywords: Sleep quality; Chronic pain; Temporomandibular disorder; Pittsburgh Sleep Quality Index; STOP-Bang; Epworth Sleepiness Scale

\section{Introduction}

Sleep maintains homeostasis and optimizes various functions across multiple physiologic systems. Good sleep quality is a key factor for good physical health, emotional well-being, brain functioning, daytime performance, and pain control. Humans require both sleep and pain for good health and survival. However, 'chronic' pain can lower and deteriorate the quality of life [1-3]. Patients with chronic pain have poorer sleep than healthy controls in terms of sleep latency, sleep efficiency, and awakenings after sleep onset. Reciprocal, bidirectional interactions exist between chronic pain and sleep disorders, deterioration in either of them can ultimately become comorbid conditions [2]. In clinical settings, sleep problems have been found to impact $88 \%$ of patients with chronic pain [4]. Contrarily, more than $40 \%$ of patients who have sleep-related problems report chronic pain [5]. The 
prevalence of chronic pain ranges from 10-40\% [6], similar to that of sleep disorders, ranging from $10-36 \%$ [7].

Temporomandibular disorders (TMDs) are quite common chronic orofacial pain conditions. TMDs are highly prevalent, affecting up to $25 \%$ of the population, with a peak incidence at 20-40 years of age, and 1.5-2 times more prevalent in female than in male [8]. TMD may promote widespread idiopathic pain, and the etiology of chronic TMD is considered multifactorial. Pain is the most common symptom of TMDs, which can affect areas such as the ears, eyes and/or throat, frequently causing neck pain and headache, and involve musculoskeletal pain, disturbances in mandibular movement, and functional impairment [9]. As the international diagnostic and classification systems for TMD, the research diagnostic criteria for TMD (RDC / TMD) proposed in 1992 is the most popular, and it has been updated to the diagnostic criteria for TMD (DC / TMD) in 2014, and has been subdivided into joint- and muscle-derived TMD pain as well as headache attributed to TMD $[10,11]$. Sleep quality in patients with TMD decreases as the number of diagnoses of painful TMD increases based on the International RDC / TMD Axis I [12]. Although several studies have been conducted on sleep quality in TMD patients [13-15], few researchers use international RDC / TMD and DC / TMD. Deterioration of sleep quality and impairment of sleep structure occur in a significant proportion in TMD patients and are thought to be a risk factor for maintaining and worsening symptoms, but their impact is not clearly known due to several methodological limitations.

Chronic TMD can present persistent, recurrent, or chronic pain associated with TMJ and/or muscles involved in the masticatory system, which leads to highly disabling. Chronic TMD is also typically related to joint dysfunctions, such as disc displacement with or without reduction [16]. Approximately $90 \%$ of TMD patients reported poor sleep quality [15]. Although the etiology underlying the connection of chronic pain and poor sleep remains largely unknown, poor sleep might contribute to TMDs aggravation. In addition, TMD patient ratings of poor sleep are associated with increased clinical pain severity and psychological distress [15]. Chronic TMD is also accompanied by pain amplification characterized by hyperalgesia, allodynia, hyperalgesia, and upregulation of the serum tonicity pathway, and sleep problems and psychological disorders are common comorbidities [17]. Sleep bruxism is considered an aggravating factor for TMD pain, but the relationship remains controversial $[18,19]$. However, there is little evidence to indicate that chronic TMD patients have poor sleep quality, and it is not known which factors clearly affect the sleep quality of chronic TMD patients.

The Pittsburgh Sleep Quality Index (PSQI) is a valid, reliable, and internationally known instrument for assessing self-perceived sleep quality [20]. Compared to objective measurements, such as polysomnography or oximetry, PSQI addresses a longer time frame and is suitable for large scale research. In addition, a comparison of validity with respiratory indices from polysomnographic recordings has been made for PSQI with some degree of success. The primary concern for many patients presenting with lowered sleep quality is excessive daytime sleepiness (EDS). There may not be an obvious cause for EDS, lowered sleep quality and insufficient sleep duration are responsible for this symptom [21]. EDS is a common symptom in sleep disorders, chronic pain, headaches, and TMD [22, 23]. The EDS status can be easily evaluated with the Epworth sleepiness scale (ESS), and $28.6 \%$ of TMD patients presented with EDS based on this questionnaire [23]. Obstructive sleep apnea (OSA) is a sleep breathing disorder characterized by repeated agitation of nocturnal breathing disruption caused by upper airway collapse, and is considered as a putative risk factor for chronic TMD [24]. For screening for OSA, the snoring, tiredness, observed apnea, high blood pressure (STOP)-BMI, age, neck circumference, and male gender (Bang) questionnaire with high reliability has been used in the clinical field [24, 25]. Therefore, it may be necessary to evaluate sleep-related factors using PSQI, STOP-Bang, and ESS simultaneously in chronic TMD patients. 
This cohort study aimed to investigate the clinical characteristics and evaluate sleep-related factors to correlate these parameters with poor sleep quality in chronic TMD patients. We described sleep quality differences between chronic TMD patients and healthy controls, investigated subgroup of TMD influences, and studied sleep quality in the full set of diagnostic DC/TMD and RDC/TMD subgroups of Axis I. To complementarily evaluate the factors related to EDS and OSA along with sleep quality obtained from PSQI, ESS and STOP-Bang questionnaire were additionally used. Chronic TMD has an idiopathic basis in which the pathophysiology mechanism is not well understood. Our findings in this study will support the usefulness of an integrated model of demographics and disease characteristics in explaining sleep quality deterioration in chronic TMD patients.

\section{Materials and Methods}

\subsection{Participants}

To investigate the research purpose, the authors designed and implemented a retrospective cohort study conducted at the Department of Orofacial Pain and Oral Medicine at Kyung Hee University Dental Hospital of Seoul. The research protocol was reviewed in compliance with the Helsinki Declaration and approved by the Institutional Review Board of the Kyung Hee University Dental Hospital (KHD IRB no. 1804-2). Written informed consent was obtained from all individual participants.

Of the patients who had visited the Department of Orofacial Pain and Oral Medicine between June 1, 2018, and November 30, 2019, the study sample was composed of chronic TMD patients according to inclusion and exclusion criteria. Inclusion criteria of chronic TMD patients were as follows: completed a set of routine TMJ assessments, as well as constructive questionnaires, no treatment of the current episode other than medication, and no history of TMD prior to the present symptoms. Patients who were pregnant or had a history of systemic osteoarthritis, rheumatoid arthritis, other connective tissue diseases, general infection, neurological/neuropathic diseases, and those under 18 years of age were excluded from the study. Healthy controls were recruited through advertisements in the hospital. Inclusion criteria of healthy controls were as follows: systemic good health, no history of macrotrauma or surgery on TMJ and neck area, no previous diagnosis of TMD, no TMD signs and symptoms, and an absence of TMD on brief clinical examination. Healthy individuals who satisfies the following inclusion criteria and exclusion, and who are sex- and age-matched with chronic TMD patients were finally selected. All individuals included in the study completed three questionnaires including PSQI, ESS, and STOPBang.

\subsection{TMD Classification and clinical Evaluation}

Clinical evaluation procedures included an oral examination, interview, panoramic radiography, and a comprehensive questionnaire in RDC/TMD criteria [26] and DC/TMD Axis I diagnostic algorithms [27] for TMD diagnoses. A subtype of diagnostics included myofascial pain, disc displacement, arthralgia using RDC/TMD, and headache attributed to TMD based on DC/TMD Axis I diagnostic algorithms.

We diagnosed patients who experienced TMJ pain for more than six months after onset as chronic TMD. When the pain persisted more than three to six months, individuals were usually considered in a chronic state [28]. The intensity of TMD pain was measured using a visual analog scale (VAS) (0-10, 10 being the worst possible pain), and symptom duration in the masticatory muscles, TMJ, and adjacent structures were reported in days. Information concerning patient demographics included age, sex, height, body weight for 
body mass index $\left(\mathrm{BMI}=\right.$ weight $/$ height $\left.^{2}\right)$, and neck circumference were collected by a research assistant.

\subsection{Sleep Quality Evaluation Using PSQI}

PSQI was employed to measure sleep quality. Habitual sleep quality and sleep disturbance in the past month were assessed using the 19-item PSQI, a well-validated self-report questionnaire. The PSQI has seven components that concern subjective sleep quality, sleep latency, sleep duration, sleep efficiency, sleep disturbances, use of sleep medication, and daytime dysfunction. Each subscale is weighted equally, scored from 0 (good sleep/no problems) to 3 (poor sleep/severe problems), summing to a global PSQI score (range, 021). Higher scores denote worse sleep quality, and a global score $>5$ has a diagnostic value in distinguishing poor from good sleep [29]. In this study, the authors investigated sleep quality using PSQI in multidimensional construction.

\subsection{The risk evaluation of obstructive sleep apnea (OSA) with STOP-Bang}

The STOP-Bang questionnaire is validated screening tool for identifying high likelihood for OSA. The STOP-Bang questionnaire includes eight dichotomous (yes/no) questions related to these clinical features of sleep apnea. For each question, answering "yes" scores 1 , a "no" response scores 0 , and the total score ranges from 0-8. The exposure of interest was a binary-low or high likelihood for OSA; the low likelihood of OSA: Yes to $<3$ questions, high likelihood of OSA: Yes to $\geq 3$ questions [30]. All patients were asked to complete the STOP-Bang questionnaire.

\subsection{Excessive daytime sleepiness measured by ESS}

The ESS is a validated clinical tool for the evaluation of excessive daytime sleepiness (EDS) [31]. Unlike other scales, which measure sleepiness at a single time point, the ESS is designed to evaluate the general level of sleepiness. The ESS is an eight-item, self-administered questionnaire designed to provide a measure of the subject's propensity to fall asleep in a variety of situations. The subject is instructed to answer how likely it is that he/she would fall asleep in those different situations, by giving a score on a 4-point scale (0-3). Thus, the total score (the sum of scores of the eight items) of the ESS ranges from 024. The higher the score, the greater the possibility the individual will fall asleep during the daytime. The ESS total scores were dichotomized into scores $\leq 10$ and $>10$; the latter is considered to be clinically significant EDS [32]. We used a score of 10 or higher on the ESS to measure excessive sleepiness.

\subsection{Statistical Analysis}

The data were analyzed using SPSS Statistics for Windows, Version 20.0 (IBM Corp., Armonk, NY, USA). Continuous variables are presented as means and standard deviations (SD), and categorical variables are presented as frequencies and percentages. Differences between groups were examined by using the chi-square test for categorical variables and t-test and one-way analysis of variance (ANOVA) with Tukey post-hoc test for numeric variables. Three questionnaires included in this study were for sleep quality, risk for OSA, and daytime sleepiness. To understand the risk for poor sleep quality, we performed a multiple logistic regression analysis to determine the relative risk for poor sleep (PSQI global score of $\geq 5$ ). In addition, dichotomous variables based on cut-off points of each questionnaire's global scores were used as dependent or independent variables of logistic regression analysis. The high likelihood of OSA (STOP-Bang total score $\geq 3$ ), and EDS (ESS score of $>10$ ) (independent variables), as well as age, sex, and symptom duration, were taken into consideration simultaneously to predict a value of a dependent variable (poor sleep) for chronic TMD patients. For all analyses, a two-tailed level of statistical significance of a p-value was set at less than 0.05 . 


\section{Results}

\subsection{General description}

In this period, 525 patients who were diagnosed with chronic TMD were included, and 22 patients were excluded because they lacked medical documentations. Finally, 503 patients (mean age: $33.10 \pm 13.26$ years, 333 females) were designated as the chronic TMD group. One hundred and eighty age- (mean age: $32.77 \pm 12.95$ years, 116 females) and sexmatched TMD-free volunteers were designated to the healthy control group.

Table 1 presents the demographic characteristics of chronic TMD patients compared to healthy controls. Chronic TMD was more prevalent in females $(66.2 \%)$ than in males $(43.8 \%)$, and the female:male was 1.51:1. BMI scores were significantly higher in chronic TMD than in healthy controls ( $22.32 \pm 3.57$ vs. $21.45 \pm 2.76, p=0.001)$, and the mean values were in the normal range. In chronic TMD patients, the mean VAS score of chronic TMD patients was $4.89 \pm 2.45$, and the mean symptom duration was $589.74 \pm 1315.01$ days.

\subsection{Differences in PSQI Global Scores and Poor sleep Between TMD group and Controls}

Table 2 presents the results of the subjective sleep quality in terms of PSQI score and their comparison of PSQI between chronic TMD patients and healthy controls. PSQI components, including subjective sleep quality, sleep latency, sleep duration, sleep efficiency, sleep disturbances, and use of sleep medication, were significantly higher in chronic TMD patients than in healthy controls (all p < 0.05). Interestingly, the PSQI global scores were significantly higher in chronic TMD patients than in healthy controls $(6.25 \pm 2.77 \mathrm{vs}$. 3.84 $\pm 2.29, \mathrm{p}<0.001)$. In other words, subjective sleep quality of the chronic TMD group was more impaired than the healthy control group. The proportion of poor sleepers was significantly higher in chronic TMD patients than in healthy controls $(56.9 \%$ vs. $22.2 \%$, p < 0.001 ). A total of $56.9 \%$ of chronic TMD patients were poor sleepers, and the prevalence was significantly higher in chronic TMD patients than in controls $(22.2 \%)(\mathrm{p}<0.001)$.

\subsection{Disease characteristics and their relationship with PSQI in chronic TMD patients}

Table 3 summarizes the clinical factors and subgroups of TMD diagnoses associated with impaired sleep quality. For the chronic TMD patients, both the PSQI global score (6.56 \pm 2.79 vs. $5.65 \pm 2.64, \mathrm{p}<0.001)$ and the poor sleeper ratio $(62.8 \%$ vs. $45.3 \%, \mathrm{p}<0.001)$ was significantly higher in females than in males. Conversely, in healthy control, both the PSQI global score $(3.85 \pm 2.12$ vs. $3.83 \pm 2.59, \mathrm{p}<0.944)$ and the poor sleeper ratio $(25.0 \%$ vs. $20.7 \%, \mathrm{p}=0.506$ ) were not significantly higher in females than in males.

As for age, an increase in age was associated with an increase in PSQI global scores and the occurrence of poor sleep. When we divided TMD patients into subgroups based on their age, the PSQI global scores were significantly higher and the ratio of patients with poor sleep quality was higher in patients in their $30 \mathrm{~s}$, 40s, or older as compared with those in their 20s and younger. The PSQI global score of chronic TMD patients younger than 20 years old was $4.69 \pm 2.34$, and the poor sleeper ratio was $32.8 \%$, whereas the score in patients in their 40 s and older was the highest $(7.11 \pm 2.90)$, and $71.6 \%$ of them were poor sleepers. In contrast, the PSQI global scores did not differ significant difference by age subgroup in healthy control (10s $(\mathrm{n}=20): 3.70 \pm 2.16$, $20 \mathrm{~s}(\mathrm{n}=77): 3.90 \pm 2.53,30 \mathrm{~s}(\mathrm{n}=35): 3.94$ \pm 2.41 , and over $40(\mathrm{n}=48): 3.75 \pm 1.87 ; \mathrm{p}=0.967)$. The proportion of poor sleeper also showed no significant difference by age subgroup in healthy control (10s: $20.0 \%, 20 \mathrm{~s}: 23.5 \%$, 30s: $22.9 \%$, and over $40: 20.8 \%$; $\mathrm{p}=0.905$ ).

In addition, multiple TMD sub-diagnoses were allowed in one patient, and the PSQI global score was higher when patients were diagnosed with myofascial pain (6.31 \pm 2.82 vs. $5.50 \pm 1.98, \mathrm{p}=0.023)$ or headache attributed to TMD (6.58 \pm 2.85 vs. $5.82 \pm 2.60, \mathrm{p}=$ 0.002 ) than in patients without the diagnoses. The majority of chronic TMD patients 
simultaneously suffer from pain of myofascial origin $(n=465,92.4 \%)$, arthralgia $(n=355$, $70.6 \%)$, headache attributed to TMD ( $\mathrm{n}=285,56.7 \%)$, and disc displacement $(\mathrm{n}=278$, $55.3 \%$ ). Therefore, we concluded that it is not appropriate to compare the PSQI global and component scores and presence of poor sleep by subgroup.

\subsection{Differences in STOP-Bang and ESS Between TMD group and Controls}

STOP-Bang total scores were significantly higher in TMD patients than in healthy controls $(1.77 \pm 1.86$ vs. $1.53 \pm 0.77, \mathrm{p}=0.017)$ (Table 4$)$. By detail, the proportion of snoring $(15.7 \%$ vs. $4.4 \%)$ and high blood pressure $(6.4 \%$ vs. $1.1 \%)$ were significantly higher in chronic TMD patients than in controls (all $\mathrm{p}<0.05)$. Of the 503 chronic TMD patients, $420(83.5 \%)$ had felt tired, $170(33.8 \%)$ were men, $80(15.9 \%)$ were aged over 50, $28(5.6 \%)$ had observed sleep apnea, $4(0.8 \%)$ had BMI more than $35 \mathrm{~kg} / \mathrm{m} 2$, and $29(5.8 \%)$ had larger neck circumference than the reference (male $>17$ inches, female $>16$ inches). The presence of these factors did not differ statistically from the control group. Eighty-one out of 503 chronic TMD patients (16.1\%) had a high likelihood of OSA.

Ninety-nine out of 503 chronic TMD patients (19.7\%) had EDS. Total ESS scores were not significantly different; however, the EDS rate was significantly higher in the chronic TMD group than in controls $(19.7 \%$ vs. $12.8 \%, \mathrm{p}=0.023)$.

3.5. PSQI, STOP-Bang, and ESS scores according to the number of TMD/AXIS I diagnosis When multiple diagnoses according to RDC / TMD and DC / TMD was allowed in one patient, $82.5 \%$ of chronic TMD patients had two or more multiple TMD subgroup diagnoses, and only $17.5 \%$ of the patients had one TMD diagnosis. Even, there were $33.8 \%$ (n= 170 ) of patients with four multiple diagnoses. As a result of the ANOVA test, the PSQI global score was significantly higher when chronic TMD patients had four multiple diagnoses $(6.75 \pm 2.84)$ than when they had two multiple diagnoses $(5.96 \pm 2.65)$ and three multiple diagnoses $(5.90 \pm 2.72)(\mathrm{p}=0.028)$ (Figure 1$)$. STOP-Bang total score was not significantly different according to the number of sub-diagnoses. However, in ESS, the ESS total score was significantly higher when chronic TMD patients had four multiple diagnoses $(7.40 \pm 3.79)$ than when they had only one sub-diagnosis $(5.73 \pm 3.83)(\mathrm{p}<0.001)$ (Table 5).

\subsection{Multivariate logistic regression analysis of factors influencing poor sleep quality}

Table 6 presents the significant predictors for poor sleep (PSQI > 5) among chronic TMD patients. To comprehensively examine the risk factors for a poor sleep, multivariate backward stepwise logistic regression analysis of all parameters was performed as the final analysis. The odds ratios (ORs) of each independent variable was interpreted as the change in the incidence of poor sleep in chronic TMD patients. The increase of age (OR = $2.551,95 \% \mathrm{CI}=1.662-3.917)$ was the most powerful predictor for poor sleep, and female sex $(\mathrm{OR}=1.885,95 \% \mathrm{CI}=1.193-2.978)$, ESS total score $(\mathrm{OR}=1.839,95 \% \mathrm{CI}=1.130-2.993)$, and sub-diagnosis of headache attributed to $\mathrm{TMD}(\mathrm{OR}=1.519,95 \% \mathrm{CI}=1.014-2.308)$ were putative risk factors for poor sleep. Whereas BMI, VAS, symptom duration, other subdiagnoses, except for headache, attributed to TMD, STOP-BANG total score did not reach statistical significance.

3.7. Figures and Tables

Figure 1. PSQI, STOP-Bang, and ESS scores according to number of TMD/AXIS I diagnosis. 


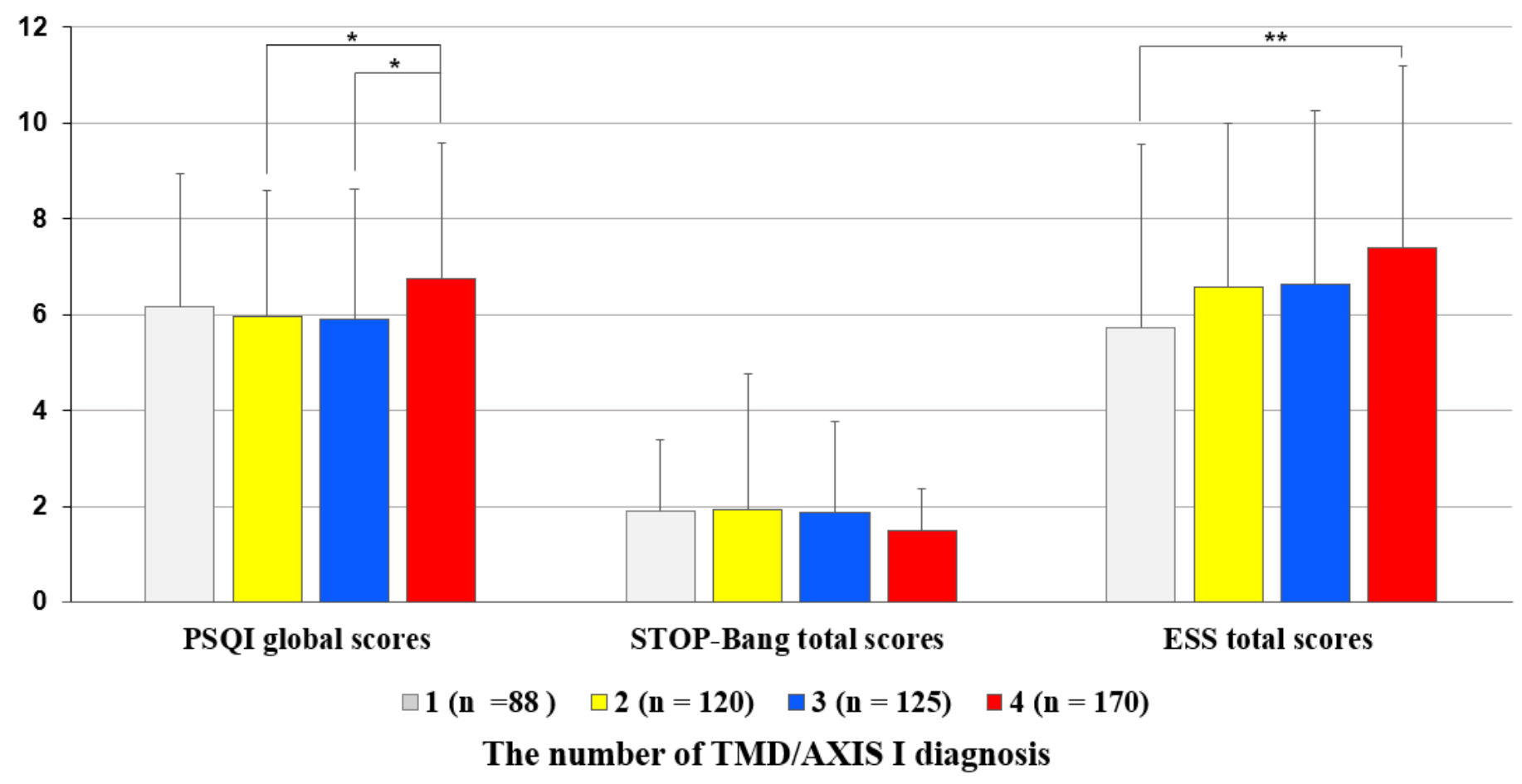

Subtypes of TMD diagnosis included myofascial pain, disc displacement, arthralgia using, and headache attributed to TMD. When multiple diagnoses were allowed for each patient, the number of diagnoses the patient had was expressed numerically. 1: In the case of having only one diagnosis among the four diagnoses of TMD based on TMD Axis I (myofascial pain, disc dis-placement, arthralgia, and headache attributed to TMD), 2: having two of the four diagnoses, 3: having three of the four diagnoses, and 4: having all of the four diagnoses. The results were obtained from ANOVA and post-hoc analysis. ${ }^{*}$ : p-value $<0.05,{ }^{* *}$ : p-value $<0.01$.

Table 1. Demographic characteristics of chronic TMD patients compared to healthy controls.

\begin{tabular}{|c|c|c|c|}
\hline Parameter & $\begin{array}{c}\text { Chronic TMD patients }(n=503) \\
n(\%) \text { or Mean } \pm \text { SD } \\
\end{array}$ & $\begin{array}{c}\text { Healthy control }(n=180) \\
n(\%) \text { or Mean } \pm \text { SD }\end{array}$ & p-value \\
\hline $\operatorname{Age~}\left(\right.$ years) $^{a}$ & $33.10 \pm 13.26$ & $32.77 \pm 12.95$ & 0.771 \\
\hline \multicolumn{4}{|l|}{ Age groups } \\
\hline Under 20 years old (10s) $\mathbf{b}$ & $61(17.59 \pm 2.28)$ & $20(17.60 \pm 1.56)$ & \\
\hline $20-30$ years old (20s) & $208(24.58 \pm 2.86)$ & $77(24.43 \pm 2.61)$ & $20 s<$ \\
\hline $31-40$ years old (30s) & $93(34.46 \pm 3.09)$ & $35(34.03 \pm 3.00)$ & $\begin{array}{l}s<0 v e r \\
40\end{array}$ \\
\hline More than 40 years old (over 40 ) & $141(51.49 \pm 7.41)$ & $48(51.55 \pm 6.87)$ & \\
\hline Sex $\left(\right.$ Female \%) ${ }^{c}$ & $333(66.2)$ & $116(64.4)$ & 0.367 \\
\hline$\overline{B M I}\left(\mathrm{~kg} / \mathrm{m}^{2}\right)^{\mathrm{a}}$ & $22.32 \pm 3.57$ & $21.45 \pm 2.76$ & $0.001 * *$ \\
\hline VAS & $4.89 \pm 2.45$ & - & n.a. \\
\hline Symptom duration (days) & $589.74 \pm 1315.01$ & - & n.a. \\
\hline
\end{tabular}

a: The results were obtained via t- test, ${ }^{\mathbf{b}}$ : The mean difference among age subgroups was obtained by one-way analysis of variance and post-hoc analysis. ${ }^{c}$ : The results were obtained from $\chi 2$ test. A p-value $<0.05$ was considered significant. **: $\mathrm{p}$ value <0.01. ***: p-value <0.001. TMD: temporomandibular disorder, BMI: body mass index, SD: standard deviation, n.a.: not available.

Table 2. Comparison of Pittsburgh Sleep Quality Index (PSQI) between chronic TMD patients and healthy controls.

\begin{tabular}{|c|c|c|c|}
\hline Parameter & $\begin{array}{c}\text { Chronic TMD patients }(\mathbf{n}=503) \\
n(\%) \text { or Mean } \pm \text { SD }\end{array}$ & $\begin{array}{c}\text { Healthy control }(n=180) \\
n(\%) \text { or Mean } \pm \text { SD }\end{array}$ & p-value \\
\hline \multicolumn{4}{|l|}{ PSQI } \\
\hline Component 1: Subjective sleep quality (0-3) ${ }^{a}$ & $1.50 \pm 0.79$ & $0.59 \pm 0.61$ & $<0.001 * * *$ \\
\hline Component 2: Sleep latency $(0-3)^{a}$ & $0.83 \pm 0.97$ & $0.42 \pm 0.72$ & $<0.001 * * *$ \\
\hline
\end{tabular}


Component 3: Sleep duration (0-3) ${ }^{\text {a }}$

$0.72 \pm 0.91$

$0.61 \pm 0.79$

0.035*

Component 4: Sleep efficiency $(0-3){ }^{\text {a }}$

$0.38 \pm 0.79$

$0.11 \pm 0.36$

$<0.001 * * *$

Component 5: Sleep disturbances (0-3) ${ }^{\text {a }}$

$1.20 \pm 0.63$

$0.45 \pm 0.60$

$<0.001 * * *$

Component 6: Use of sleep medication $(0-3)^{\text {a }}$

$0.15 \pm 0.54$

$0.04 \pm 0.28$

$0.001 * *$

Component 7: Daytime dysfunction $(0-3)^{\text {a }}$

PSQI global score (0-21) ${ }^{\mathrm{a}}$

$1.41 \pm 0.90$

$1.79 \pm 1.04$

$<0.001 * * *$

Poor sleeper (PSQI global score $\geq 5$ ) ${ }^{\text {b }}$

$6.25 \pm 2.77$

$3.84 \pm 2.29$

$<0.001 * * *$

a: The results were obtained via t- test. ${ }^{\text {b: }}$ The results were obtained from $\chi 2$ test.

A p-value $<0.05$ was considered significant. $*$ : p-value $<0.05, * *$ : p-value $<0.01$, ***: p-value $<0.001$.

TMD, temporomandibular disorder; SD, standard deviation.

Table 3. Descriptive statistics of disease characteristics and their relationship with Pittsburgh Sleep Quality Index (PSQI) in chronic TMD patients.

\begin{tabular}{|c|c|c|c|c|c|}
\hline \multirow[b]{2}{*}{ Parameter } & \multirow[b]{2}{*}{$\begin{array}{l}\text { Subgroup } \\
\text { n (\%) }\end{array}$} & \multicolumn{2}{|c|}{ PSQI } & \multicolumn{2}{|c|}{ Poor sleepers (PSQI >5) } \\
\hline & & PSQI global score & p-value ${ }^{\S}$ & $\begin{array}{c}\text { Poor sleeper } \\
\text { n }(\%)\end{array}$ & p-value ${ }^{\mathrm{II}}$ \\
\hline \multicolumn{6}{|c|}{ Demographics of TMD patients $(n=503)$} \\
\hline \multicolumn{6}{|l|}{ Age groups } \\
\hline Under 20 years old (10s) & $61(12.1)$ & $4.69 \pm 2.34$ & $<0.001 * * *$ & $20 / 61(32.8)$ & $<0.001 * * *$ \\
\hline 20-30 years old (20s) & $208(41.4)$ & $5.85 \pm 2.45$ & $10 \mathrm{~s}<20 \mathrm{~s}=30 \mathrm{~s}=$ over $40^{\dagger}$ & $104 / 208(50.0)$ & \\
\hline $31-40$ years old (30s) & $93(18.5)$ & $6.87 \pm 2.91$ & $20 \mathrm{~s}<30 \mathrm{~s}=$ over $40^{*}$ & $61 / 93(65.6)$ & \\
\hline More than 40 years old (over 40 ) & $141(28.0)$ & $7.11 \pm 2.90$ & & $101 / 141(71.6)$ & \\
\hline
\end{tabular}

Sex

\begin{tabular}{|c|c|c|c|c|c|}
\hline Male & $170(33.8)$ & $5.65 \pm 2.64$ & $<0.001 * * *$ & $77 / 170(45.3)$ & $<0.001 * * *$ \\
\hline Female & $333(66.2)$ & $6.56 \pm 2.79$ & & $209 / 333(62.8)$ & \\
\hline
\end{tabular}

TMD Axis I diagnosis in TMD patients (Multiple diagnoses are allowed per patient)

\section{Myofascial pain}

[presence]

[absence]

465 (92.4)

38 (7.6)

278 (55.3)

225 (44.7)

$6.27 \pm 2.77$

0.862

$159 / 278(57.2)$

0.928

[absence]

Arthralgia
[presence]
[absence]

355 (70.6)

148 (29.4)

$6.22 \pm 2.88$

0.888

203/355 (57.2)

0.448

Headache attributed to TMD
[presence]
285 (56.7)
$6.58 \pm 2.85$
[absence]
218 (43.3)
$5.82 \pm 2.60$

$0.002 * *$

178/285 (62.5)

$108 / 218$ (49.5)

$127 / 225$ (56.4)

\$: The $\mathrm{p}$-value for the test of the mean difference of the PSQI global score between subgroups. II: The p-value for the comparison results of the number ( $\%$ ) between subgroups via $\chi 2$ test and Boneferroni correction. The mean difference among age subgroups was obtained by one-way analysis of variance and post-hoc analysis. ${ }^{\dagger}$ : The PSQI global scores of subgroups over 20 years of age were significantly higher than that of $10 \mathrm{~s}$, and the scores of $20 \mathrm{~s}, 30 \mathrm{~s}$, and over 40 were not significantly different. $*$ : The PSQI global scores over 30 years of age were significantly higher than that of 20s, and the scores of 30 s and over 40 were not significantly different. p-Value significance was set at $<0.05$. *: p-value $<0.05$, **: p-value <0.01, ***: p-value <0.001. TMD: temporomandibular disorder, PSQI: Pittsburgh Sleep Quality Index, SD: standard deviation. 
Table 4. Comparison of STOP-BANG and ESS components between groups.

\begin{tabular}{|c|c|c|c|}
\hline Parameter & $\begin{array}{c}\text { Chronic TMD } \\
\text { patients }(n=503) \\
\text { n }(\%) \text { or Mean } \pm \text { SD }\end{array}$ & $\begin{array}{c}\text { Healthy control } \\
(\mathrm{n}=180) \\
\mathrm{n}(\%) \text { or Mean } \pm \text { SD }\end{array}$ & p-value \\
\hline \multicolumn{4}{|l|}{ STOP-Bang } \\
\hline Snoring $(\text { none }=0, \text { yes }=1)^{\mathrm{a}}$ & $79(15.7)$ & $8(4.4)$ & $0.001 * * *$ \\
\hline Tired (none $=0$, yes $=1)^{\mathrm{a}}$ & $420(83.5)$ & $147(81.7)$ & 0.324 \\
\hline Observed apnea $(\text { none }=0 \text {, yes }=1)^{\mathrm{a}}$ & $28(5.6)$ & $6(3.3)$ & 0.163 \\
\hline High blood pressure $(\text { none }=0, \text { yes }=1)^{a}$ & $32(6.4)$ & $2(1.1)$ & $0.002 * *$ \\
\hline BMI more than $35 \mathrm{~kg} / \mathrm{m}^{2}(\text { none }=0 \text {, yes }=1)^{\mathrm{a}}$ & $4(0.8)$ & $1(0.6)$ & 0.604 \\
\hline Age over $50(\text { none }=0 \text {, yes }=1)^{\mathrm{a}}$ & $80(15.9)$ & $30(16.7)$ & 0.814 \\
\hline $\begin{array}{l}\text { Neck circumference (male }>17 \text { inches, female }>16 \text { inches) } \\
(\text { none }=0, \text { yes }=1)^{\text {a }}\end{array}$ & $29(5.8)$ & $7(3.9)$ & 0.223 \\
\hline Gender, male $(\text { Female }=0, \text { male }=1)^{\mathrm{a}}$ & $170(33.8)$ & $64(35.6)$ & 0.367 \\
\hline STOP Bang total score $(0-8)$ & $1.77 \pm 1.86$ & $1.53 \pm 0.77$ & $0.017 *$ \\
\hline STOP-Bang total score $\geq 3$ (high risk of OSA) & $81(16.1)$ & $13(7.2)$ & $0.001 * *$ \\
\hline \multicolumn{4}{|l|}{ Epworth sleepiness scale } \\
\hline Sitting and reading $(0-3)^{\mathbf{b}}$ & $0.94 \pm 0.73$ & $0.91 \pm 0.64$ & 0.588 \\
\hline Watching TV $(0-3)^{\mathbf{b}}$ & $0.63 \pm 0.67$ & $0.61 \pm 0.60$ & 0.751 \\
\hline Sitting, inactive in public place $(0-3)^{b}$ & $0.59 \pm 0.66$ & $0.76 \pm 0.73$ & $0.007 * *$ \\
\hline As a passenger in a car for an hour without a break $(0-3)^{b}$ & $1.19 \pm 0.89$ & $\mathbf{1 . 0 4} \pm 0.81$ & $0.047 *$ \\
\hline Lying down to rest in the afternoon when circumstances permit $(0-3)^{b}$ & $1.45 \pm 0.89$ & $1.17 \pm 0.84$ & $\begin{array}{c}< \\
0.001 * * *\end{array}$ \\
\hline Sitting and talking to someone $(0-3)^{\mathbf{b}}$ & $0.28 \pm 0.54$ & $0.33 \pm 0.56$ & 0.272 \\
\hline Sitting quietly after a lunch without alcohol $(0-3)^{\mathbf{b}}$ & $1.23 \pm 0.85$ & $1.34 \pm 0.83$ & 0.107 \\
\hline In a car, while stopped for a few minutes for traffic $(0-3)^{b}$ & $0.42 \pm 0.63$ & $0.54 \pm 0.66$ & $\mathbf{0 . 0 4 3 *}$ \\
\hline ESS total score $(0-24)^{\mathbf{b}}$ & $6.73 \pm 3.62$ & $6.71 \pm 3.02$ & 0.952 \\
\hline ESS total score $\geq 10(\text { EDS })^{\text {a }}$ & $99(19.7)$ & $23(12.8)$ & $\mathbf{0 . 0 2 3} *$ \\
\hline
\end{tabular}

Values are presented as number (\%) and mean \pm standard deviation. ${ }^{a}$ : Comparison results of the number (\%) between groups performed with $\chi^{2}$ test and Boneferroni correction. ${ }^{\mathrm{b}}$ : The results were obtained via $\mathrm{t}-\mathrm{test}$. $\mathrm{p}$-Value significance was set at $<0.05$. *: p-value <0.05, **: p-value <0.01, ***: p-value <0.001. TMD: temporomandibular disorder, SD: standard deviation, STOP-Bang: The snoring, tiredness, observed apnea, high blood pressure (STOP)-BMI, age, neck circumference, and gender (Bang), ESS: Epworth sleepiness scale, EDS: excessive daytime sleepiness.

Table 5. PSQI, STOP-Bang, and ESS scores according to number of TMD/AXIS I diagnosis.

\begin{tabular}{lcccccc}
\hline & \multicolumn{3}{c}{ Number of TMD Axis I diagnosis } \\
Parameter & $\mathbf{1}(\mathbf{n}=\mathbf{8 8})$ & $\mathbf{2}(\mathbf{n}=\mathbf{1 2 0})$ & $\mathbf{3}(\mathbf{n}=\mathbf{1 2 5})$ & $\mathbf{4}(\mathbf{n}=\mathbf{1 7 0})$ & p-value & Post-hoc analysis \\
\hline PSQI global scores & $\mathbf{6 . 1 8} \pm \mathbf{2 . 7 7}$ & $\mathbf{5 . 9 6} \pm \mathbf{2 . 6 5}$ & $\mathbf{5 . 9 0} \pm \mathbf{2 . 7 2}$ & $\mathbf{6 . 7 5} \pm \mathbf{2 . 8 4}$ & $\mathbf{0 . 0 2 8} *$ & $\mathbf{2}<4, \mathbf{3}<\mathbf{4}$ \\
STOP-Bang total scores & $1.90 \pm 1.51$ & $1.95 \pm 2.82$ & $1.88 \pm 1.89$ & $1.49 \pm 0.87$ & 0.125 & $\mathbf{0 . 0 0 5 * *}$ \\
ESS total scores & $\mathbf{5 . 7 3} \pm \mathbf{3 . 8 3}$ & $\mathbf{6 . 5 9} \pm \mathbf{3 . 4 1}$ & $\mathbf{6 . 6 5} \pm \mathbf{3 . 6 0}$ & $\mathbf{7 . 4 0} \pm \mathbf{3 . 7 9}$ & $\mathbf{1 < 4}$ \\
\hline Var
\end{tabular}

Values are presented as mean \pm standard deviation. The results were obtained from ANOVA and post-hoc analysis. p-Value significance was set at <0.05. *: p-value <0.05, **: p-value <0.01. TMD: temporomandibular disorder, PSQI: Pittsburgh Sleep Quality Index, STOP-Bang: The snoring, tiredness, observed apnea, high blood pressure (STOP)-BMI, age, neck circumference, and gender (Bang), ESS: Epworth sleepiness scale. When multiple diagnoses were allowed for each patient, the number of diagnoses the patient had was expressed numerically. 1: In the case of having only one diagnosis among the four diagnoses of TMD based on TMD Axis I (myofascial pain, disc dis-placement, arthralgia, and headache attributed to TMD), 2: having two of the four diagnoses, 3: having three of the four diagnoses, and 4: having all of the four diagnoses.

\begin{tabular}{|c|c|c|c|c|c|c|}
\hline \multirow[b]{2}{*}{ Parameter } & \multirow{2}{*}{$\begin{array}{c}\text { PSQI }(\leq 5) \\
(n=217)\end{array}$} & \multirow{2}{*}{$\begin{array}{c}\text { PSQI }(>5) \\
(n=286)\end{array}$} & \multirow{2}{*}{ p-value } & \multirow{2}{*}{$\operatorname{Exp}(B)$} & \multicolumn{2}{|c|}{$95 \%$ Confidential interval } \\
\hline & & & & & Lower & Upper \\
\hline Age [ref.=under average value] & $56(25.8)$ & $141(49.3)$ & $<0.001 * * *$ & 2.551 & 1.662 & 3.917 \\
\hline Female $[$ ref. $=$ male $]$ & $124(57.1)$ & $209(73.1)$ & $0.007 * *$ & 1.885 & 1.193 & 2.978 \\
\hline BMI [ref.= under average value $]$ & $90(41.5)$ & $139(48.6)$ & 0.146 & 1.353 & 0.900 & 2.036 \\
\hline VAS [ref.=under average value] & $121(55.8)$ & $168(58.7)$ & 0.951 & 1.012 & 0.685 & 1.495 \\
\hline Duration [ref.=under average value] & $61(28.1)$ & $73(25.5)$ & 0.711 & 0.922 & 0.600 & 1.418 \\
\hline Myofascial pain by RDC/TMD [ref.=none] & $197(90.8)$ & $268(93.7)$ & 0.762 & 1.125 & 0.523 & 2.423 \\
\hline Disc displacement by RDC/TMD [ref.=none] & $119(54.8)$ & $159(55.6)$ & 0.453 & 0.829 & 0.508 & 1.353 \\
\hline Arthralgia by RDC/TMD [ref.=none] & $152(70.0)$ & $203(71.0)$ & 0.930 & 0.976 & 0.576 & 1.655 \\
\hline
\end{tabular}




\begin{tabular}{lcccccc} 
Headache attributed to TMD by DC/TMD & $\mathbf{1 0 7}(\mathbf{4 9 . 3})$ & $\mathbf{1 7 8}(\mathbf{6 2 . 2})$ & $\mathbf{0 . 0 4 9} *$ & $\mathbf{1 . 5 1 9}$ & $\mathbf{1 . 0 0 0}$ & $\mathbf{2 . 3 0 8}$ \\
STOP-Bang [ref.<3] & $31(14.3)$ & $50(17.5)$ & 0.868 & 1.051 & 0.584 & 1.891 \\
ESS [ref.< 10] & $\mathbf{3 4}(\mathbf{1 5 . 7})$ & $\mathbf{6 5}(\mathbf{2 2 . 7})$ & $\mathbf{0 . 0 1 4}$ & $\mathbf{1 . 8 3 9}$ & $\mathbf{1 . 1 3 0}$ & $\mathbf{2 . 9 9 3}$ \\
\hline
\end{tabular}

Table 6. Multivariate logistic regression analysis of factors influencing poor sleep (PSQI > 5) among the TMD.

OR: odds ratio, CI: Confidential interval, ESS: Epworth sleepiness scale. Multivariate logistic regression analysis was performed to comprehensively examine the factors affecting poor sleep in chronic TMD patients $(\mathrm{R}=0.833, \mathrm{R}$ square $=0.787)$.

For obtaining significant results, two-tailed level of statistical significance of a p-value was set at less than 0.05 .

\section{Discussion}

This study comprehensively investigated the clinical characteristics and sleep quality of chronic TMD patients who were diagnosed based on RDC/TMD and DC/TMD Axis I. The main findings in the present study imply that chronic state TMD patients had poorer sleep than healthy controls, and the magnitude of impaired sleep was associated with increased age, female sex, certain subtypes of TMD diagnosis, including myofascial pain and headache attributed to TMD, and the number of TMD diagnoses in a person. Regarding summary scores and cut-off values of each questionnaire, the presence of EDS was a significant predictor for the poor sleep quality in chronic TMD patients.

The cause of chronic TMD is varied, and its localization, and clinical characteristics are vaguer than with acute pain. Chronic TMD is typically associated with joint dysfunctions such as disc displacement with or without reduction [16], and psychological distress [17]. It is difficult to infer a causal relation between sleep and chronic pain; patients with chronic pain commonly suffer from poor sleep quality [33]. Approximately $45.5 \%$ of patients with chronic pain suffer from sleep disorders, and older age was significantly associated with pain experience [7]. In this study, $56.9 \%$ of the chronic TMD group met the proposed cut-off of 5 of PSQI for poor sleep, compared to $22.2 \%$ of the control group that had impaired sleep. The proportion of TMD patients with poor sleep (56.9\%) in the present study is higher than the rate reported among adults with TMD $(43.3 \%)$ in other studies $[34,35]$ but lower than those in other studies involving older TMD patients (69.6-90.0\%) $[15,36]$.

The etiology of chronic TMD fundamentally related to peripheral and central factors together. Peripheral factors of TMD include inflammatory processes, including synovitis and myositis, infection, or irritation. Peripheral factors are the main cause of acute pain, but as the pain becomes chronic, the importance of the central factor increases [37]. Central factors include sleep deterioration, impairment of psychological health, and dysfunction of central pain inhibitory system [38, 39]. Furthermore, central sensitization is a key characteristic of chronic pain presented as hypersensitivity, particularly tactile allodynia, hyperalgesia, and enhanced temporal summation [40,41], which commonly presents in chronic TMD patients. As in other idiopathic pain disorders such as fibromyalgia and irritable bowel syndrome, TMD patients frequently present with overlapping signs and symptoms of sleep disorders [42].

Sleep quality of chronic TMD patients was more impaired by increased age. In the present study, the increase of age (OR $=2.551,95 \% \mathrm{CI}=1.662-3.917)$ was the most powerful predictor for poor sleep, and female sex $(\mathrm{OR}=1.885,95 \% \mathrm{CI}=1.193-2.978)$ was followed. In young adults, consolidated sleep at night and wakefulness during the day emerges from a balance between the brainstem, hypothalamus, and midbrain [43]. In older adults, this operation is not effective, and decreased sleep duration, increased sleep latency, impaired sleep quality, shallow sleep, and changes in sleep structure can lead to sustained or deepening pain [44]. In addition, sleep patterns and structures are known to change across the lifespan, with up to $50 \%$ of older adults report difficulties initiating and/or maintaining sleep [45]. Chronic sleep disturbances are considered as indications of poor health, vice versa, older adults commonly suffer from pain syndromes, arthritis, hormonal changes, 
neurodegeneration, psychological distress, cancer, renal and urologic diseases, and medical comorbidities all of which can contribute to sleep disorders [46]. Thus, older adults with chronic TMD are less likely to get enough rest and recovery through sleep than younger ones.

Female sex was also a major contributor to poor sleep quality in chronic TMD patients. There is limited recent evidence of interactions among sex, TMD chronicity, and sleep. However, it has been found that females show higher clinical and experimental pain sensitivity, and worse sleep impairments than males [2]. Few probable causes for poor sleep quality in the female sex may be explained based on sex differences concerning mechanisms of pain of the craniofacial system [47]. Furthermore, contribution of female sex may reflect changes of systems beyond the physical axis of the orofacial area and in line with the biopsychosocial model, blending centrally mediated factors. Especially in postmenopausal female, an increase in sleep problems may be associated with the presence of noticeable hormonal changes, age-associated changes in sleep and psychosocial distress [2]. In clinical research, females reported TMD symptoms, headache, and had muscle tenderness and joint sounds more often than males [48]. It will be crucial to determine whether the effect of sleep on chronic TMD pain, and vice versa, is moderated by key demographic variables, such as age or sex.

Considering the EDS, EDS was a significant predictor for poor sleep in chronic TMD patients. EDS was more prevalent in chronic TMD patients than in healthy controls $(19.7 \%$ vs. $12.8 \%, \mathrm{p}<0.05)$, and its OR value for poor sleep quality was 1.069 . The EDS prevalence in the present study was higher than the prevalence among the general population (12$16 \%$ ) [49], and lower than $28.57 \%$ of TMD patients, the rate previously reported [23]. The discrepancy may have occurred due to differences in age distribution, race, and method of study. A significant proportion of the general population, as well as in patients with sleep problems or chronic pain, may suffer from EDS for a variety of causes, not pathological mechanisms. In older adults, they are prone to have daytime napping and EDS and the presence of comorbid conditions such as chronic pain, sleep disorders, and frequent nighttime urination breaks [50]. Diminished melatonin secretion and a reduced circadian modulation of rapid-eye-movement sleep and less pronounced day-night differences in the lower alpha activity occurs in the older group [51]. Furthermore, females are more likely than males to have more trouble sleeping at night and experience EDS [52]. Thus, EDS in chronic TMD may have different underlying mechanisms of a homeostatic drive for sleep and pain control systems according to age and sex. Hence, it is necessary to consider age-and sex-related differences in chronic TMD patients to obtain accurate results translation.

The high likelihood of OSA was not the significant predictor for poor sleep quality in chronic TMD patients. The STOP-Bang questionnaire is a great tool to easily identify patients with suspected OSA [25]. However, traditionally, polysomnography (PSG) in a sleep laboratory has been considered as a standard tool for the diagnosis of OSA [53]. Once OSA is identified, these patients need a definite diagnosis using the PSG. There is a limit to the extended interpretation of the results of the sleep quality and high risk of OSA in patients with chronic TMD, as the relationship between OSA and TMD. Of course, a bidirectional association between OSA and TMD have been suggested [24, 54]. Although the clear mechanism has not been identified, oral appliances used in the treatment of OSA may be the cause of TMD with continuous mandibular advancement while sleeping [55]. Sleep bruxism, a major risk factor for TMD, may be linked to OSA through the sleeprelated arousal reactions [56]. The link between sleep bruxism and OSA has been studied at various ages [57-59], which could potentially suggest a link between TMD and OSA. Sleep bruxism and PSG were not covered in this study, but will be fully addressed in subsequent studies. 
Headache attributed to TMD was associated with an increase of PSQI global scores, and a significant predictor for poor sleep. As headaches are a common symptom of accompanying TMD, few researches have been done on the nature of headache attributed to chronic TMD. TMD patients with headache reported significantly higher levels of pain and mandibular dysfunction than patients with only TMD [60]. Headache can promote sleep disturbances, and sleep disturbances can also precede or trigger a headache attack [61]. Moreover, sleep deterioration has been associated with an increased risk for headaches, and in individuals with chronic headaches, shorter sleep duration has been associated with more severe pain [62]. Clearly, the underlying pathophysiology contributing to the close association and complex relationship among headache attributed to TMD, headache disorders, and sleep disorders are not fully explained. There may be complex bidirectional relationships, and can be explained by peripheral and central sensitization, malfunctions of neuroendocrine, immune, and vascular system, and even gene polymorphism.

Myofascial pain was also associated with poor sleep quality in chronic TMD patients. Similar to the present study, the substantial influence of myofascial pain on poor sleep quality in patients with TMD was documented [63]. Furthermore, according to the TMD diagnostic subgroups, and the impact on quality of sleep and the symptom severity can vary. TMD patients with muscle-derived or myofascial pain exhibit more advanced stages of depression and somatization than patients diagnosed with TMJ disc displacement, a jointderived problem [64]. Other researchers also observed a higher impact in patients with myogenous complaints than those with disc disorders $[65,66]$. While joint pain is characterized by a well-defined inflammatory process, chronic muscle pain presents with enigmatic pathophysiologic mechanisms, of which central sensitization is the common factor unifying these conditions [67]. In addition, females have more pain and widespread pain in more body areas than males, which may be related to their worse quality of sleep [68]. Therefore, depending on the subgroup of TMD, the mechanisms by which TMD signs and symptoms occur are different, and further investigation is needed on the effects on sleep quality.

Finally, the quality of sleep was lower in chronic TMD patients with multiple diagnoses than in patients with a single diagnosis. According to Gil-Martínez et al., patients with mixed pain, having arthrogenous and myogenous origin simultaneously, showed greater craniomandibular and neck disability than patients diagnosed with chronic joint pain or muscle pain only [69]. Patients with headache and TMDs reported significantly higher levels of pain and disability compared to patients with only TMDs [60]. These findings can be interpreted as increasing the number of TMD subgroup diagnoses can increase the severity of TMD symptoms. Overall, chronic TMD symptoms and multiple diagnoses may have a bidirectional cross-correlation, which can impair sleep quality. Chronic TMD, especially myofascial pain, headaches attributed to TMD, and sleep disturbance factors, may share the mechanism of occurrence and exacerbation.

Limitations of this study include the case-control study design, which cannot address a causal direction of effects and suggests only associations/correlations between the variables. A study design with repeated PSG may be more powerful in detecting phase-related differences. Instead of using PSG, we used self-assessment measures of sleep due to feasibility and convenience, especially because of large sample size. PSG is an objective measure of biophysiologic sleep parameters, so we are planning further studies to expand these findings with PSG. In addition, to get a deeper understanding of the relation between chronic TMD and poor sleep quality, we need to investigate their biopsychosocial aspects; however, this study did not evaluate psychological distress in patients with chronic TMD. Further studies on the psychological aspects of chronic TMD patients are ongoing. 


\section{Conclusions}

The strength of this study is a comprehensive analysis of how various issues affect poor sleep quality among many factors. Chronic TMD patients suffered more from impaired sleep than healthy control subjects, and poor sleep was associated with multiple comorbid symptoms. Thus, assessing sleep quality should be a routine part of the diagnostic workup of chronic TMD patients. Furthermore, a multidisciplinary management approach is needed to address all the factors in addition to sleep that modulate pain experience. In the diagnosis and treatment of chronic TMD, a fragmented field of view is not suitable, and a multidisciplinary approach involving experts in neurology, endocrinology, gerontology, and psychology, in addition to orofacial pain experts, is required. The results from this study will help to establish strategies for individual treatment and management of chronic TMD patients.

\section{Supplementary Materials: None.}

Author Contributions: Y.-H.L. wrote the paper. Y.-H.L. and Q.-S.A. contributed to data acquisition, Y.-H.L., and J.-S.A. to both data analysis and interpretation. Y.-H.L., Q.-S.A., and J.-S.A. provided their expertise and contributed to the figures. Y.-H.L. and T.K. provided their expertise and contributed to revisions. All authors have read and agreed to the published version of the manuscript.

Funding: This research was supported by the National Research Foundation of Korea Grant (NRF/2020R1F1A1070072) funded by the Korean government which was funded by Y.-H.L.

Institutional Review Board Statement: The research protocol was reviewed in compliance with the Helsinki Declaration and approved by the Institutional Review Board of the Kyung Hee University Dental Hospital (KHD IRB no. 1804-2).

Informed Consent Statement: Informed consent was obtained from all subjects involved in the study.

Data Availability Statement: Since these are patient data, if there is a request for data disclosure, KHU-IRB will discuss it before disclosure.

Acknowledgments: None.

Conflicts of Interest: The authors declare no conflict of interest. 


\section{References}

1. Worley SL: The Extraordinary Importance of Sleep: The Detrimental Effects of Inadequate Sleep on Health and Public Safety Drive an Explosion of Sleep Research. P T 2018, 43(12):758-763.

2. Finan PH, Goodin BR, Smith MT: The association of sleep and pain: an update and a path forward. J Pain 2013, 14(12):15391552.

3. Dueñas M, Ojeda B, Salazar A, Mico JA, Failde I: A review of chronic pain impact on patients, their social environment and the health care system. J Pain Res 2016, 9:457-467.

4. Smith MT, Perlis ML, Smith MS, Giles DE, Carmody TP: Sleep quality and presleep arousal in chronic pain. Journal of behavioral medicine 2000, 23(1):1-13.

5. Ohayon MM: Relationship between chronic painful physical condition and insomnia. Journal of psychiatric research 2005, 39(2):151-159.

6. Nickel R, Raspe HH: [Chronic pain: epidemiology and health care utilization]. Der Nervenarzt 2001, 72(12):897-906.

7. Jank R, Gallee A, Boeckle M, Fiegl S, Pieh C: Chronic Pain and Sleep Disorders in Primary Care. Pain Res Treat 2017, 2017:9081802-9081802.

8. Warren MP, Fried JL: Temporomandibular disorders and hormones in women. Cells Tissues Organs 2001, 169(3):187-192.

9. Suvinen TI, Reade PC, Kemppainen P, Könönen M, Dworkin SF: Review of aetiological concepts of temporomandibular pain disorders: towards a biopsychosocial model for integration of physical disorder factors with psychological and psychosocial illness impact factors. Eur J Pain 2005, 9(6):613-633.

10. Anderson GC, Gonzalez YM, Ohrbach R, Truelove EL, Sommers E, Look JO, Schiffman EL: The Research Diagnostic Criteria for Temporomandibular Disorders. VI: future directions. Journal of orofacial pain 2010, 24(1):79-88.

11. Schiffman E, Ohrbach R, Truelove E, Look J, Anderson G, Goulet J-P, List T, Svensson P, Gonzalez Y, Lobbezoo F et al: Diagnostic Criteria for Temporomandibular Disorders (DC/TMD) for Clinical and Research Applications: recommendations of the International RDC/TMD Consortium Network* and Orofacial Pain Special Interest Groupt. J Oral Facial Pain Headache 2014, 28(1):6-27.

12. Rener-Sitar K, John MT, Pusalavidyasagar SS, Bandyopadhyay D, Schiffman EL: Sleep quality in temporomandibular disorder cases. Sleep Med 2016, 25:105-112.

13. Porto F, de Leeuw R, Evans DR, Carlson CR, Yepes JF, Branscum A, Okeson JP: Differences in psychosocial functioning and sleep quality between idiopathic continuous orofacial neuropathic pain patients and chronic masticatory muscle pain patients. Journal of orofacial pain 2011, 25(2):117-124.

14. Schmitter M, Kares-Vrincianu A, Kares H, Bermejo JL, Schindler HJ: Sleep-associated aspects of myofascial pain in the orofacial area among Temporomandibular Disorder patients and controls. Sleep medicine 2015, 16(9):1056-1061.

15. Yatani H, Studts J, Cordova M, Carlson CR, Okeson JP: Comparison of sleep quality and clinical and psychologic characteristics in patients with temporomandibular disorders. Journal of orofacial pain 2002, 16(3):221-228.

16. Graff-Radford SB: Temporomandibular disorders and headache. Dent Clin North Am 2007, 51(1):129-144, vi-vii.

17. Maísa Soares G, Rizzatti-Barbosa CM: Chronicity factors of temporomandibular disorders: a critical review of the literature. Braz Oral Res 2015, 29.

18. Lobbezoo F, Lavigne GJ: Do bruxism and temporomandibular disorders have a cause-and-effect relationship? J Orofac Pain 1997, 11(1):15-23.

19. Manfredini D, Lobbezoo F: Relationship between bruxism and temporomandibular disorders: a systematic review of literature from 1998 to 2008. Oral Surg Oral Med Oral Pathol Oral Radiol Endod 2010, 109(6):e26-50.

20. de Tommaso M, Delussi M, Vecchio E, Sciruicchio V, Invitto S, Livrea P: Sleep features and central sensitization symptoms in primary headache patients. J Headache Pain 2014, 15(1):64-64.

21. Slater G, Steier J: Excessive daytime sleepiness in sleep disorders. J Thorac Dis 2012, 4(6):608-616. 
22. Kristoffersen ES, Stavem K, Lundqvist C, Russell MB: Excessive daytime sleepiness in secondary chronic headache from the general population. The Journal of Headache and Pain 2017, 18(1):85.

23. Jariyasakulroj S, Mitrirattanakul S: Excessive daytime sleepiness in temporomandibular disorder patients. Cranio 2020, 38(3):144-148.

24. Sanders AE, Essick GK, Fillingim R, Knott C, Ohrbach R, Greenspan JD, Diatchenko L, Maixner W, Dubner R, Bair E et al: Sleep apnea symptoms and risk of temporomandibular disorder: OPPERA cohort. J Dent Res 2013, 92(7 Suppl):70s-77s. Chung F, Abdullah HR, Liao P: STOP-Bang Questionnaire: A Practical Approach to Screen for Obstructive Sleep Apnea. Chest 2016, 149(3):631-638.

26. Manfredini D, Segu M, Bertacci A, Binotti G, Bosco M: Diagnosis of temporomandibular disorders according to RDC/TMD axis I findings, a multicenter Italian study. Minerva stomatologica 2004, 53(7-8):429-438.

Schiffman E, Ohrbach R, Truelove E, Look J, Anderson G, Goulet JP, List T, Svensson P, Gonzalez Y, Lobbezoo F et al: Diagnostic Criteria for Temporomandibular Disorders (DC/TMD) for Clinical and Research Applications: recommendations of the International RDC/TMD Consortium Network* and Orofacial Pain Special Interest Groupdagger. J Oral Facial Pain Headache 2014, 28(1):6-27.

Treede R-D, Rief W, Barke A, Aziz Q, Bennett MI, Benoliel R, Cohen M, Evers S, Finnerup NB, First MB et al: A classification of chronic pain for ICD-11. Pain 2015, 156(6):1003-1007.

Van Ravesteyn LM, Tulen JHM, Kamperman AM, Raats ME, Schneider AJT, Birnie E, Steegers EAP, Hoogendijk WJG, Tiemeier HW, Lambregtse-van den Berg MP: Perceived sleep quality is worse than objective parameters of sleep in pregnant women with a mental disorder. Journal of clinical sleep medicine : JCSM : official publication of the American Academy of Sleep Medicine 2014, 10(10):1137-1141.

30. Chung F, Subramanyam R, Liao P, Sasaki E, Shapiro C, Sun Y: High STOP-Bang score indicates a high probability of obstructive sleep apnoea. British journal of anaesthesia 2012, 108(5):768-775.

31. Boyes J, Drakatos P, Jarrold I, Smith J, Steier J: The use of an online Epworth Sleepiness Scale to assess excessive daytime sleepiness. Sleep E breathing = Schlaf E Atmung 2017, 21(2):333-340. Sayar K, Arikan M, Yontem T: Sleep quality in chronic pain patients. Can J Psychiatry 2002, 47(9):844-848.

Smith MT, Wickwire EM, Grace EG, Edwards RR, Buenaver LF, Peterson S, Klick B, Haythornthwaite JA: Sleep disorders and their association with laboratory pain sensitivity in temporomandibular joint disorder. Sleep 2009, 32(6):779-790.

Benoliel R, Zini A, Zakuto A, Slutzky H, Haviv Y, Sharav Y, Almoznino G: Subjective Sleep Quality in Temporomandibular Disorder Patients and Association with Disease Characteristics and Oral Health-Related Quality of Life. J Oral Facial Pain Headache 2017, 31(4):313-322.

Natu VP, Yap AU, Su MH, Irfan Ali NM, Ansari A: Temporomandibular disorder symptoms and their association with quality of life, emotional states and sleep quality in South-East Asian youths. Journal of oral rehabilitation 2018, 45(10):756763.

Crofford LJ: Chronic Pain: Where the Body Meets the Brain. Trans Am Clin Climatol Assoc 2015, 126:167-183.

Gil-Martínez A, Paris-Alemany A, López-de-Uralde-Villanueva I, La Touche R: Management of pain in patients with temporomandibular disorder (TMD): challenges and solutions. J Pain Res 2018, 11:571-587. Smith MT, Wickwire EM, Grace EG, Edwards RR, Buenaver LF, Peterson S, Klick B, Haythornthwaite JA: Sleep disorders and their association with laboratory pain sensitivity in temporomandibular joint disorder. Sleep 2009, 32(6):779-790. 
43. Blumberg MS, Gall AJ, Todd WD: The development of sleep-wake rhythms and the search for elemental circuits in the infant brain. Behav Neurosci 2014, 128(3):250-263.

44. Gori S, Lucchesi C, Morelli N, Maestri M, Bonanni E, Murri L: Sleep-related migraine occurrence increases with aging. Acta neurologica Belgica 2012, 112(2):183-187.

45. Inouye SK, Studenski S, Tinetti ME, Kuchel GA: Geriatric syndromes: clinical, research, and policy implications of a core geriatric concept. Journal of the American Geriatrics Society 2007, 55(5):780-791.

46. Mander BA, Winer JR, Walker MP: Sleep and Human Aging. Neuron 2017, 94(1):19-36.

47. Dao TT, LeResche L: Gender differences in pain. Journal of orofacial pain 2000, 14(3):169-184; discussion 184-195.

48. Magnusson T, Egermark I, Carlsson GE: A longitudinal epidemiologic study of signs and symptoms of temporomandibular disorders from 15 to 35 years of age. Journal of orofacial pain 2000, 14(4):310-319.

49. Roth T, Roehrs TA: Etiologies and sequelae of excessive daytime sleepiness. Clinical therapeutics 1996, 18(4):562-576; discussion 561 .

50. Stepnowsky CJ, Ancoli-Israel S: Sleep and Its Disorders in Seniors. Sleep Med Clin 2008, 3(2):281-293.

51. Münch M, Knoblauch V, Blatter K, Schröder C, Schnitzler C, Kräuchi K, Wirz-Justice A, Cajochen C: Age-related attenuation of the evening circadian arousal signal in humans. Neurobiol Aging 2005, 26(9):1307-1319.

Boccabella A, Malouf J: How Do Sleep-Related Health Problems Affect Functional Status According to Sex? J Clin Sleep Med 2017, 13(5):685-692.

Kapur VK, Auckley DH, Chowdhuri S, Kuhlmann DC, Mehra R, Ramar K, Harrod CG: Clinical Practice Guideline for Diagnostic Testing for Adult Obstructive Sleep Apnea: An American Academy of Sleep Medicine Clinical Practice Guideline. J Clin Sleep Med 2017, 13(3):479-504.

54. Wu JH, Lee KT, Kuo CY, Cheng CH, Chiu JY, Hung JY, Hsu CY, Tsai MJ: The Association between Temporomandibular Disorder and Sleep Apnea-A Nationwide Population-Based Cohort Study. Int J Environ Res Public Health 2020, 17(17).

Näpänkangas R, Raunio A, Sipilä K, Raustia A: Effect of mandibular advancement device therapy on the signs and symptoms of temporomandibular disorders. J Oral Maxillofac Res 2013, 3(4):e5-e5.

Wieckiewicz M, Bogunia-Kubik K, Mazur G, Danel D, Smardz J, Wojakowska A, Poreba R, Dratwa M, ChaszczewskaMarkowska M, Winocur E et al: Genetic basis of sleep bruxism and sleep apnea-response to a medical puzzle. Sci Rep 2020, 10(1):7497.

Jokubauskas L, Baltrušaitytė A: Relationship between obstructive sleep apnoea syndrome and sleep bruxism: a systematic review. J Oral Rehabil 2017, 44(2):144-153.

58. Guo H, Wang T, Li X, Ma Q, Niu X, Qiu J: What sleep behaviors are associated with bruxism in children? A systematic review and meta-analysis. Sleep Breath 2017, 21(4):1013-1023.

59. Castroflorio T, Bargellini A, Rossini G, Cugliari G, Deregibus A: Sleep bruxism in adolescents: a systematic literature review of related risk factors. Eur J Orthod 2017, 39(1):61-68.

60. Di Paolo C, D'Urso A, Papi P, Di Sabato F, Rosella D, Pompa G, Polimeni A: Temporomandibular Disorders and Headache: A Retrospective Analysis of 1198 Patients. Pain Res Manag 2017, 2017:3203027-3203027.

61. Fernandez-de-Las-Penas C, Fernandez-Munoz JJ, Palacios-Cena M, Paras-Bravo P, Cigaran-Mendez M, Navarro-Pardo E: Sleep disturbances in tension-type headache and migraine. Therapeutic advances in neurological disorders 2018, 11:1756285617745444.

Houle TT, Butschek RA, Turner DP, Smitherman TA, Rains JC, Penzien DB: Stress and sleep duration predict headache severity in chronic headache sufferers. Pain 2012, 153(12):2432-2440.

63. Vazquez-Delgado E, Schmidt JE, Carlson CR, DeLeeuw R, Okeson JP: Psychological and sleep quality differences between chronic daily headache and temporomandibular disorders patients. Cephalalgia : an international journal of headache 2004, 24(6):446-454. 
64. Ferrando M, Andreu Y, Galdón MJ, Durá E, Poveda R, Bagán JV: Psychological variables and temporomandibular disorders: distress, coping, and personality. Oral Surg Oral Med Oral Pathol Oral Radiol Endod 2004, 98(2):153-160.

65. Bush FM, Harkins SW: Pain-related limitation in activities of daily living in patients with chronic orofacial pain: psychometric properties of a disability index. Journal of orofacial pain 1995, 9(1):57-63.

66. Auerbach SM, Laskin DM, Frantsve LM, Orr T: Depression, pain, exposure to stressful life events, and long-term outcomes in temporomandibular disorder patients. Journal of oral and maxillofacial surgery : official journal of the American Association of Oral and Maxillofacial Surgeons 2001, 59(6):628-633; discussion 634.

67. Furquim BDA, Flamengui LMSP, Conti PCR: TMD and chronic pain: a current view. Dental Press J Orthod 2015, 20(1):127133.

68. Bartley EJ, Fillingim RB: Sex differences in pain: a brief review of clinical and experimental findings. Br J Anaesth 2013 , 111(1):52-58.

69. Gil-Martínez A, Grande-Alonso M, López-de-Uralde-Villanueva I, López-López A, Fernández-Carnero J, La Touche R: 